\title{
A Novel Routing Efficient Algorithm Based on Clustering in WSNs
}

\author{
Fakhrosadat Fanian ${ }^{1 *}$ and Marjan Kuchaki Rafsanjani ${ }^{2}$ \\ 'Department of Computer, Science and Research Branch, Islamic Azad University, Kerman, Iran; \\ fanianfakhri@yahoo.com \\ 2Department of Computer Science, Shahid Bahonar University of Kerman, Kerman, Iran; \\ kuchaki@uk.ac.ir
}

\begin{abstract}
Application of Wireless Sensor Networks becomes important nowadays. One of the efficient and prevalent usages of Wireless Sensor Networks is using clustering in such a network. In fact, sensor nodes might reduce overload communications. With this purpose, it was tried to deliver an algorithm which prevents direct connection between all cluster heads and base station; instead, cluster heads should use other cluster heads near to the base station in order to transfer data to the base station; they were selected based on the factors such as cluster head distance with base station and distance among cluster heads. Also, in this proposed algorithm, routes are selected such that, the selected routes' length is almost equal and finally results in work load balance among cluster heads and decrease energy consumptions and increase network lifetime. In addition, time delay for data transfer to base station becomes leved off in all routes.
\end{abstract}

Keywords: Wireless Sensor Network (WSN), Clustering, Routing.

\section{Introduction}

Wireless Sensor Networks includes numerous sensor nodes which are responsible to collect and transfer data [1]. Network data transmissions consume most energy [2]. Clustering trend is based on routing, which might be efficient in reduction of energy consumption and communications [1-5].

Many algorithms and protocols were introduced in this area with the attempt to decrease energy consumption and increase network lifetime. But in most cases, what ignored was on how to transfer data from cluster heads to base station. Usually the data is transferred to base station directly after data collection. The base station can be located in the vicinity [6-10] or to be addressed from cluster heads distance to base station necessitating the optimization of the distance [11-13]. Earlier, more complicated algorithms, ignoring route distances, were used in hierarchical transfer of data among cluster heads.

In this section, briefly, we review some of clustering methods to clarify the proposed algorithm aspects that are introduced in the next section. One of these methods is PEGASIS [14]. In fact, this protocol transfers data to the base station using chain production mechanisms of sensor nodes and connection of each sensor node just with its adjacent node. This chain is based on base station data or with the help of nodes, so that the remotest sensor nodes send a powerful signal. Based on received response it calculates adjacent nodes and then set its signal power. So that, just one of the adjacent nodes might receive its powerful signal; this process continues to the point so that the chain forms and each sensor node in the chain

*Corresponding author:

Fakhrosadat Fanian (fanianfakhri@yahoo.com) 
combined its data with received data from all other nodes and forwarded them to reach base station [15].

After PEGASIS Protocol introduction, increased delay issue was seen during data transfer to base station; Two plans have been proposed in order to solve this problem [16]. One of the plans used sensor nodes CDMA capabilities and the other ignored this capability. Both methods attempt to determine routes with least delay to send and receive data using a binary composition plan with parallel communications.

In HCC [17] Protocol, the structure of sensor node clustering forms in multi layers. Here, the node with least ID obtains priority to start clustering and if most of the nodes desire to make cluster formation process, they must perform two work steps of tree discovery. In some methods [18], the node was used with rich source that usually selected as cluster heads and otherwise it was played Gateway role and transfer data from cluster heads to base station.

A protocol was introduced, which allowed cluster heads in multi-hop form to transfer their data to the base station through middle cluster heads, when cluster heads' distance from the base station is quite long. [19] This method tried to select a route with least multi-hop using flooding method. Another method was introduced, [20] which produced multi routes among cluster heads, than select an optimal route among them. In fact, each cluster head prefers to send its data to adjacent cluster head and not directly to the base station.

Three layered routing was done by Zhixiang and Bensheng [21] to communicate multi-hop among cluster heads. Initially, based on probability, first layer cluster heads were selected. Among first layer cluster heads, second layer cluster heads were determined based on energy factor. Data are forwarded to first layer cluster heads after collection and then forwarded to second layer cluster heads and finally sent to the base station.

In PECRP [22], data transfer was performed as multihop by selection of cluster heads near to them. In such methods data are received from several cluster heads nearer to it and routes are not separated and also the routes distances vary from one another.

In this study, we introduce a simple, new algorithm to decrease cluster heads' energy consumption and reduce delay in data transfer to base station.

The rest of this article is organized as follows: Section 2 introduces proposed network model. Section 3 describes proposed algorithm in details, and finally Section 4 concludes the article.

\section{Network Model}

A study was performed, on how a data collection functions' in periodic mode in homogenous wireless sensor network which includes many sensor nodes with limited energy. In this function, sensor nodes in periodic form sent their data to cluster heads, which are responsible for data collection and combination. Supposed hypothesizes are as follows:

1. All sensors are remote from the base station and scattered evenly in random style.

2. All sensors and base station are still and immobile.

3. All sensors contain same initial energy and each sensor with sufficient energy might connect and communicate with base station and each node knows the base station place.

4. Each cluster head knows its situation.

\section{Proposed Algorithm}

In this algorithm, the base station function is to compute and determine several distinct and separate routes. They have almost equal lengths among cluster heads in order to decrease energy consumption. It reduces delay in data transfer to the main station and makes balance workloads of clusters.

In other words, data routing was performed by the base station. A message is sent to cluster heads. In this algorithm, base station is based on an interval table made by the base station at the initial stage of each period. The base station determines routes based on regulations come in the next part. It also sends data cluster heads. In fact, by this way, it was determined to collect data movement route toward itself.

Base stations send a message to cluster heads in order to identify cluster head situation. Each cluster head which receives this message is again sent to base station. Then the base station becomes aware of all cluster heads situation. According to equation 1, base station produces interval table between each cluster heads and itself and distance of each cluster heads with other cluster heads. In equation $1, d(i, j)$ is the distance between two cluster heads of $\mathrm{CH}_{i}$ and $\mathrm{CH}_{j}$ with $\left(x_{i}, y_{i}\right)$ and $\left(x_{j}, y_{j}\right)$ coordinates.

$$
d(i, j)=\sqrt{\left(x_{j}-x_{i}\right)^{2}+\left(y_{j}-y_{i}\right)^{2}}
$$


After data formation, for instance, for cluster heads with specification in Table 1, interval table produces base station as shown in Table 2. Regulation is used by the base station in order to determine separate routes and multi-hop. They have equal lengths. Initially, there are two options:

1. Since, lower interval limit of cluster heads divide by two, it was determined that cluster head numbers have the longest distance to the base station. In the proposed algorithm, they are the requirements at research start (in above mentioned example it was selected two cluster heads of $\mathrm{CH}_{4}, \mathrm{CH}_{5}$ ).

2. Due to lower interval limit of cluster heads divide by two, it was determined cluster head numbers with the shortest distance to the base station. In the proposed algorithm, they are the requirement of research end (in above mentioned example it was selected two cluster heads of $\mathrm{CH}_{1}, \mathrm{CH}_{2}$ ). In order to select middle cluster heads, we address as follows:

- If the cluster heads are equal to zero, data of those cluster heads forwarded to base station directly. If they are equal to 1 , it was considered two, sharing in selection of start and end points of search ignored.

- Always from remote cluster heads to base station search was started in interval table.

- Nodes are the start requirements of search. They receive pockets contained address, if selected during the search in interval table was ignored.

- Cluster head selected from interval table for a route if selected for future searches it was ignored.

- The end requirement of research is reaching to cluster heads determined as the end requirements. If one of these cluster heads selected by a route, in following routes we must reach another cluster head as end requirements of search.

Table 1. Show Cartesian coordinates of $\mathrm{CHs}$ and $\mathrm{BS}$

\begin{tabular}{cll}
\hline $\mathrm{Y}$ & $\mathrm{X}$ & \\
\hline 11 & 5 & $\mathrm{BS}$ \\
9.5 & 2 & $\mathrm{CH}_{1}$ \\
8.1 & 6.7 & $\mathrm{CH}_{2}$ \\
6.3 & 3.5 & $\mathrm{CH}_{3}$ \\
5 & 5.7 & $\mathrm{CH}_{4}$ \\
3.1 & 8.7 & $\mathrm{CH}_{5}$ \\
\hline
\end{tabular}

Table 2. Length difference between base station and cluster heads and distance among cluster heads

\begin{tabular}{lllllll}
\hline & BS & $\mathrm{CH}_{1}$ & $\mathrm{CH}_{2}$ & $\mathrm{CH}_{3}$ & $\mathrm{CH}_{4}$ & $\mathrm{CH}_{5}$ \\
\hline $\mathrm{BS}$ & 0 & 3.35 & 3.36 & 4.93 & 6.04 & 8.72 \\
$\mathrm{CH}_{1}$ & 3.35 & 0 & 4.90 & 3.53 & 5.82 & 9.26 \\
$\mathrm{CH}_{2}$ & 3.36 & 4.90 & 0 & 3.67 & 3.25 & 5.38 \\
$\mathrm{CH}_{3}$ & 4.93 & 3.53 & 3.67 & 0 & 2.55 & 6.10 \\
$\mathrm{CH}_{4}$ & 6.04 & 5.82 & 3.25 & 2.55 & 0 & 3.55 \\
$\mathrm{CH}_{5}$ & 8.72 & 9.26 & 5.38 & 6.10 & 3.55 & 0 \\
\hline
\end{tabular}

According to the above mentioned regulations, two routes were determined by the base station and sent to $\mathrm{CH}_{4}, \mathrm{CH}_{5}$ cluster heads.

The first route is the route that the base station sends its data to $\mathrm{CH}_{5}$.

$$
\mathrm{CH}_{5} \rightarrow \mathrm{CH}_{2} \rightarrow \text { Base station }
$$

The second route is the one that the base station sends its data to $\mathrm{CH}_{4}$.

$$
\mathrm{CH}_{4} \rightarrow \mathrm{CH}_{3} \rightarrow \mathrm{CH}_{1} \rightarrow \text { Base station }
$$

According to Table 2, the lengths of route for both routes are as follows:

First route length: $5.38+3.36=8.74$

Second route length: $2.55+3.53+3.35=9.43$

It can be seen that route lengths are almost equal.

\section{Conclusion}

In this article, we introduce an algorithm in addition to multi-hop communication between cluster heads. Also, it might be useful in clustering trend according to balance cluster heads load. Also, contrary to other methods of multi-hop, it never imposed overload on a cluster head near to base station. It tried to distribute workload on several cluster heads. Due to creation of the same length of these routes, results in delay of data arrival to the base station are equal for all routes. Generally, this algorithm results in a decrease in energy consumption and finally increases network lifetime. 


\section{References}

1. Akyildiz I F, Su W et al. (2002). Wireless sensor networks: a survey, Computer Networks, vol 38(4), 393-422.

2. Akyildiz I F, Su W et al. (2002). A survey on sensor networks, IEEE Communications Magazine, vol 40(8), 102-114.

3. Akkaya K, and Younis M (2005). A survey on routing protocols for wireless sensor networks, Ad Hoc Networks, vol 3(3), 325-349.

4. Rajavivarma V, Yang Y et al. (2003). An overview of wireless sensor networks and applications, Proceedings of the 35th IEEE Southeastern Symposium on System Theory (SSST), 432-436.

5. Chong C Y, and Kumar S P (2003). Sensor networks: evolution, opportunities, and challenges, Proceedings of the IEEE, vol 91(8), 1247-1256.

6. Heinzelman W R, Chandrakasan A et al. (2000). Energy-efficient communication protocol for wireless microsensor networks, Proceedings of the IEEE International Conference on System Sciences (ICSS), 1-10.

7. Younis O, and Fahmy S (2004). HEED: a hybrid, energyefficient, distributed clustering approach for ad hoc sensor networks, IEEE Transactions on Mobile Computing, vol 3(4), 366-379.

8. Wang A, Yang D et al. (2011). A clustering algorithm based on energy information and cluster heads expectation for wireless sensor networks, Computers and Electrical Engineering, vol 38(3), 662-671.

9. Hong J, Kook G et al. (2009). T-LEACH: the method of threshold-based cluster head replacement for wireless sensor networks, Information Systems Frontiers, vol 11(5), 513-521.

10. Bagherzadeh J, and Samadzamini M (2009). A clustering algorithm for wireless sensor networks based on density of sensors, Proceedings of the 7th International Conference on Mobile Computing and Multimedia (MoMM), 594-598.

11. Ye M, Li C et al. (2005). An energy efficient clustering scheme in wireless sensor networks, Proceedings of the 24th IEEE International Performance Computing and Communications Conference (IPCCC), 535-540.

12. Xunbo L, Na L et al. (2010). An improved LEACH for clustering protocols in wireless sensor networks, Proceedings of the IEEE International Conference on Measuring
Technology and Mechatronics Automation (ICMTMA), 496-499.

13. Min X, Wei-Ren S et al. (2010). Energy efficient clustering algorithm for maximizing lifetime of wireless sensor networks, Electronics and Communication, vol 64(4), 289-298.

14. Lindsey S, and Raghavendra C S (2002). PEGASIS: power efficient gathering in sensor information systems, Proceedings of the IEEE Aerospace Conference, 1125-1130.

15. Lindsey S, and Raghavendra C S et al. (2000). Data gathering in sensor networks using the energy delay metric, Proceedings of the 15th IEEE International Parallel and Distributed Processing Symposium (IPDPS), 2001-2008.

16. Lindsey S, and Raghavendra C S et al. (2002). Data gathering algorithms in sensor networks using energy metrics, IEEE Transactions on Parallel and Distributed Systems, vol 13(9), 924-935.

17. Banerjee S, and Khuller S (2001). A clustering scheme for hierarchical control in multihop wireless networks, Proceedings of the 20th Joint Conference of the Computer and Communications Societies (INFOCOM'01), IEEE, vol 2, 1028-1037.

18. Abdellah E, Benalla $S$ et al. (2010). Advanced low energy adaptive clustering hierarchy, Proceedings of the International Journal on Computer Science and Engineering (IJCSE), vol 2(7), 2491-2497.

19. Biradar R V, Sawant S R et al. (2011). Multihop routing in self-organizing wireless sensor networks, International Journal of Computer Science Issues (IJCSI), vol 8(1), 155-164.

20. Xiangning F, and Yulin S (2007). Improvement on LEACH protocol of wireless sensor network, Proceedings of the IEEE International Conference on Sensor Technologies and Applications (SENSORCOMM), 260-264.

21. Zhixiang D, and Bensheng Q (2007). Three-layered routing protocol for WSN based on LEACH algorithm, Proceedings of the IEEE Communications conference on Wireless, Mobile and Sensor Networks (CCWMSN), 72-75.

22. Liu T, and Li F (2009). Power-efficient clustering routing protocol based on applications in wireless sensor network, Proceedings of the 5th IEEE International Conference on Wireless Communications Networking and Mobile Computing (WiCOM), 1-6. 